\title{
Cicatrização de ferida cirúrgica tratada com laser de baixa intensidade: relato de caso
}

Healing of surgical wound treated with low-level laser: case report

Curación de heridas quirúrgicas tratadas con láser de bajo nivel: reporte de caso

Matheus Francisco Barros RODRIGUES ${ }^{1}$

Layla Louise de Amorim ROCHA ${ }^{1}$

Iana Maria Gomes BARBOSA ${ }^{1}$

Rodrigo da Franca ACIOLY2

Daniel do Carmo CARVALHO ${ }^{2}$

Cristofe Coelho Lopes da ROCHA ${ }^{3}$

Maria Cátia Mendes Rodrigues GONÇALVES ${ }^{3}$

${ }^{I}$ Curso de Odontologia, Faculdade Cathedral, 69307-053 Boa Vista - RR, Brasil

${ }^{2}$ Departamento de Cirurgia e Traumatologia Bucomaxilofacial, Hospital Geral de Roraima, 69305-455 Boa Vista - RR, Brasil

${ }^{3}$ Departamento infraestrutura, Instituto Federal, 69303-340 Boa Vista - RR, Brasil

${ }^{3}$ Departamento de Medicina, Universidade Federal de Roraima, 69310-000 Boa Vista - RR, Brasil

\section{Resumo}

Os lasers de baixa potência são utilizados para fins terapêuticos e bioestimuladores, agindo principalmente como aceleradores em processos cicatriciais, além de possuírem efeitos redutores de dor e anti-inflamatórios. A cicatrização envolve fenômenos biológicos como alterações vasculares e celulares, proliferação epitelial, proliferação de fibroblastos, revascularização e contração da ferida. Em tecidos epiteliais, o laser causa a proliferação, migração de células e ativa os fatores de crescimento. Já em tecido conjuntivo, atua aumentando a síntese de colágeno por fibroblastos, além de aumentar a vascularização ao promover angiogênese. Este artigo tem por objetivo relatar um caso de exposição óssea pós-cirúrgica tratada com terapia a laser de baixa intensidade, demonstrando aspectos clínicos e teóricos, além de analisar os efeitos da laserterapia e sua importância no processo de cicatrização. Em casos de exposição óssea uma alternativa viável é a enxertia de tecido gengival. No entanto, a necessidade de dois procedimentos cirúrgicos na área doadora e da receptora pode apresentar-se como desvantagem na aplicabilidade da técnica. Considera-se que a laserterapia possibilita a regeneração com ausência de procedimentos cirúrgicos, além de promover o aumento do fluxo sanguíneo e controle de processos inflamatórios. Pode-se concluir que a laserterapia de baixa intensidade representa um auxílio no processo de cicatrização e possibilitou a resolução do caso clínico de maneira eficaz.

Descritores: Lasers; Odontologia; Cicatrização.

\section{Abstract}

Low-power lasers are used for therapeutic and biostimulating purposes, acting mainly as accelerators in healing processes, in addition to having pain-reducing and anti-inflammatory effects. Healing involves biological phenomena such as vascular and cellular changes, epithelial proliferation, fibroblast proliferation, revascularization and wound contraction. In epithelial tissues, laser causes proliferation, cell migration and activates growth factors, whereas in connective tissue, it acts increasing the synthesis of collagen by fibroblasts, in addition to increasing vascularization by promoting angiogenesis. This article aims to report a case of post-surgical bone exposure treated with low-level laser therapy, demonstrating clinical and theoretical aspects, in addition to analyzing the effects of laser therapy, as well as its importance in the healing process. In cases of bone exposure, a viable alternative is gingival tissue grafting. However, the need for two surgical procedures in the donor site and recipient area can pose a disadvantage in the applicability of the technique. It is considered that laser therapy allows regeneration without surgical procedures, in addition to promoting increased blood flow and control of inflammatory processes. It can be concluded that low-level laser therapy represents an aid in the healing process, and it enabled the resolution of the clinical case in an effective manner.

Descriptors: Lasers; Dentistry; Wound Healing,

\section{Resumen}

Los láseres de baja potencia se utilizan con fines terapéuticos y bioestimuladores, actuando principalmente como aceleradores en los procesos de curación, además de tener efectos antiinflamatorios y reductores del dolor. La curación involucra fenómenos biológicos tales como cambios vasculares y celulares, proliferación epitelial, proliferación de fibroblastos, revascularización y contracción de la herida. En los tejidos epiteliales, el láser causa proliferación, migración celular y activa factores de crecimiento. Ya en el tejido conectivo, actúa aumentando la síntesis de colágeno por los fibroblastos, además de aumentar la vascularización al promover la angiogénesis. Este artículo tiene como objetivo informar un caso de exposición ósea posquirúrgica tratada con terapia con láser de bajo nivel, demostrando aspectos clínicos y teóricos, además de analizar los efectos de la terapia con láser y su importancia en el proceso de curación. En casos de exposición ósea, una alternativa viable es el injerto de tejido gingival. Sin embargo, la necesidad de dos procedimientos quirúrgicos en el área donante y receptora puede presentar una desventaja en la aplicabilidad de la técnica. Se considera que la terapia con láser permite la regeneración en ausencia de procedimientos quirúrgicos, además de promover un mayor flujo sanguíneo y el control de los procesos inflamatorios. Se puede concluir que la terapia con láser de bajo nivel representa una ayuda en el proceso de curación y permitió la resolución del caso clínico de manera efectiva.

Descriptores: Rayos Láser; Odontología; Cicatrización de Heridas.

\section{INTRODUÇÃO}

O laser interage com a matéria viva por meio dos processos ópticos de reflexão, transmissão, espalhamento e absorção. Quando incide no tecido biológico, parte da luz não penetra, sendo refletida. A porção de luz que penetra no tecido será dividida em uma parte que será absorvida, outra parte que será espalhada e ainda outra que será transmitida ${ }^{1}$.

Segundo Cavalcanti et al. ${ }^{1}$, os lasers podem ser classificados em: (i) lasers de alta potência chamados lasers cirúrgicos ou HILT (high intensity laser treatment) que possuem indicações cirúrgicas de corte, coagulação e cauterização com efeitos de ablação; e (ii) lasers de baixa potência chamados lasers terapêuticos ou LILT (low intensity laser therapy) que são utilizados para fins terapêuticos e bioestimuladores, agindo principalmente como aceleradores em processos cicatriciais.

$\mathrm{O}$ crescente uso de terapia a laser de baixo e alto nível se dá principalmente devido a grande influencia que esse método exerce nos tecidos 2 . Os principais resultados da laserterapia são redução da dor, ação anti-inflamatória, ${ }^{3,4}$ além do efeito fotobiológico que promove aceleração dos eventos do processo de cicatrização tecidual ${ }^{4}$. 
A cicatrização envolve fenômenos biológicos como alterações vasculares e celulares, proliferação epitelial, proliferação de fibroblastos, revascularização e contração da ferida ${ }^{4}$. Uma ferida é caracterizada pela interrupção na continuidade de um tecido corporal, podendo ser causada por traumas físicos, químicos e mecânicos ou até mesmo desencadeada por uma condição médica ${ }^{5}$.

A utilização dos lasers pode diferir quanto à potência e dose utilizada e quanto ao modo e número de aplicações. O aumento do número de aplicações promove aceleração do processo de cicatrização tecidual $^{4}$. A terapia possui metodologia simples, natureza localizada e não invasiva podendo estar associada à maioria dos tratamentos odontológicos ${ }^{6}$.

Em tecidos epiteliais o laser causa a proliferação, migração e adesão de células e ativa os fatores de crescimento ${ }^{7}$. Entretanto, em tecido conjuntivo atua aumentando a síntese de colágeno por fibroblastos, além de aumentar a vascularização ao promover angiogênese ${ }^{7}$.

Este artigo tem por objetivo relatar um caso de exposição óssea pós-cirúrgica tratada com terapia a laser de baixa intensidade, demonstrando aspectos clínicos e teóricos, além de analisar os efeitos da laserterapia, bem como sua importância no processo de cicatrização.

\section{CASO CLÍNICO}

Paciente do sexo feminino, 44 anos, procurou o consultório odontológico relatando dores em função da dificuldade de cicatrização, após ter sido submetida a procedimento cirúrgico para remoção de tórus mandibular há cerca de 15 dias. Durante a anamnese e exame clínico foi observado exposição óssea no sítio cirúrgico em ambos os lados da região óssea lingual de mandíbula, como pode ser observado na Figura 1.

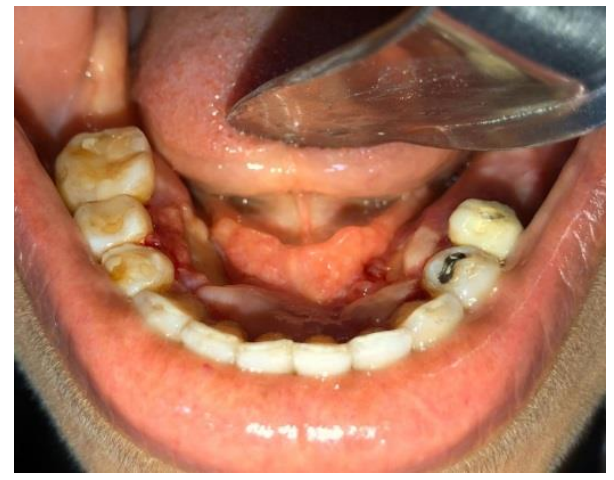

Figura 1: Exposição óssea em região lingual.

A terapia a laser de baixa intensidade foi instituída como tratamento principal, com frequência diária por um período de 3 semanas, utilizando o equipamento Therapy XT. Aliado à laserterapia foi realizado desgaste superficial com broca cirúrgica a fim de estimular o sangramento da região, visando contribuir no processo de cicatrização. Na primeira semana de tratamento foi observado melhoria, com recobrimento por tecido mole de parte do osso exposto, conforme Figura 2.

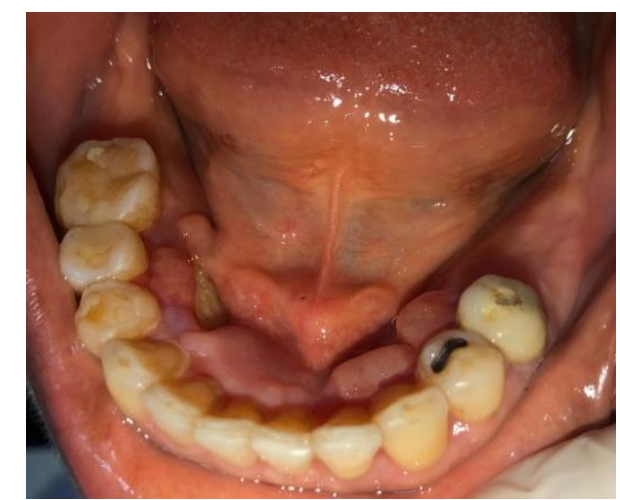

Figura 2: Ferida cirúrgica no sétimo dia de terapia a laser.

Paciente respondeu bem ao tratamento $\mathrm{e}$ obteve êxito no fechamento da ferida cirúrgica, como pode ser visto na Figura 3, não havendo presença de sinais flogísticos.

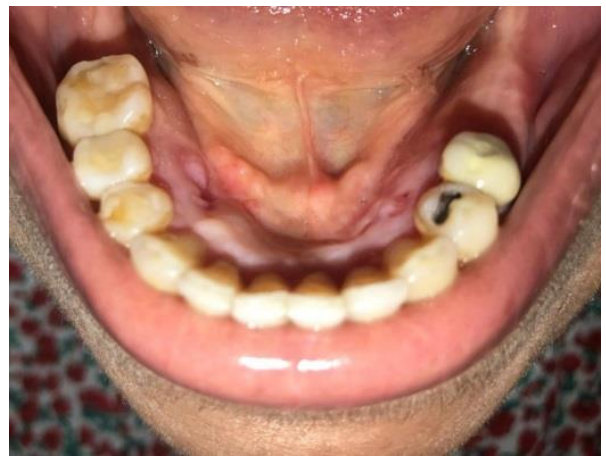

Figura 3: Fechamento da ferida cirúrgica na terceira semana de laserterapia.

\section{DISCUSSÃO}

Traumas e lesões fazem com que o corpo humano emita uma sequência de respostas bioquímicas e celulares com a intenção de restaurar a integridade do tecido envolvido e a capacidade funcional ${ }^{8}$. Porém, o atraso ou deficiência nesse processo pode acarretar necrose, quadros de infecção e sensibilidade ${ }^{9}$. Portanto, o cirurgião deve estabelecer a terapêutica adequada visando otimizar o processo de reparo, levando em consideração os aspectos dos distintos procedimentos.

A enxertia de tecido gengival representa uma alternativa viável em casos de grandes áreas de reabsorção do tecido com exposição óssea, radicular e de implantes ${ }^{10}$. No entanto, a necessidade de dois procedimentos cirúrgicos na área doadora e da receptora pode significar uma desvantagem na aplicabilidade da técnica ${ }^{11}$.

O processo de reparo tecidual é complexo e compreende alterações vasculares e celulares, proliferação epitelial e de fibroblastos ${ }^{12}$. Porém a terapia a laser possibilita a regeneração com ausência de procedimentos cirúrgicos, além de promover o aumento do fluxo sanguíneo e controle de processos inflamatórios ${ }^{3}$. Entretanto, é importante considerar que diferentes reações teciduais podem ser obtidas na 
terapia com lasers de baixa potência, dependendo da idade, sexo e fatores de nutrição tecidual e sistêmica ${ }^{13}$.

\section{CONSIDERAÇÕES FINAIS}

Pode-se concluir que o tratamento por meio da laserterapia de baixa intensidade representa um auxílio na otimização do processo de cicatrização. Sua ação proporciona a liberação de fatores de crescimento, melhoria no fluxo sanguíneo e controle de processos inflamatórios ${ }^{1,4,7}$, além de dispensar intervenção cirúrgica. A terapia a laser possibilitou resolução do caso clínico de maneira eficaz, em um período de três semanas, com ausência de queixas álgicas ou processos infecciosos.

\section{REFERÊNCIAS}

1. Cavalcanti TM, Almeida-Barros RQ, Catão MHCV, Feitosa APA, Lins RDAU. Conhecimento das propriedades físicas e da interação do laser com os tecidos biológicos na odontologia. An Bras Dermatol. 2011;86(5):955-60.

2. Saracino S, Mozzati M, Martinasso G, Poi R, Canuto RA, Muzio G. Superpulsed laser irradiation increases osteoblast activity via modulation of bone morphogenetic factors. Lasers Surg Med. 2009;41(4):298-304.

3. Aykol G, Baser U, Maden I, Kazak Z, Onan U et al. The effect of low-level laser therapy as an adjunct to non-surgical periodontal treatment. J Periodontol. 2011;82(3):481-88.

4. Bourguignon-Filho AM, Feitosa ACR, Beltrão GC, Pagnoncelli RM. Utilização do laser de baixa intensidade no processo de cicatrização tecidual. Revisão da literatura. Rev Port Estomatol Med Dent Cir Maxilofac. 2005;46(1):37-43.

5. Chaves MEA, Araújo AR, Piancastelli ACC, Pinotti M. Effects of low-power light therapy on wound healing: LASER $x$ LED. An Bras Dermatol. 2014;89(4):616-23.

6. Assis, VKS, Cardoso FL, Silva BP. Aplicabilidade da laserterapia no cenário odontológico: uma terapêutica em ascensãorevisão de literatura. Anais do Seminário Científico do UNIFACIG 5;2019.

7. Silva Garcez A, Simões Ribeiro M, Núñez SC. Laser de baixa potência: princípios básicos e aplicações clínicas na Odontologia. Rio de Janeiro: Elsevier; 2012.

8. Miloro M. Princípios de cirurgia bucomaxilofacial de Peterson. 2. ed. São Paulo: Santos; 2008.

9. Laureano A, Rodrigues AM. Cicatrização de feridas. Rev. da Soc. Port. Dermatologia e Venereol. 2011;69(3):355-67.

10. Carvalho LMM, Guimarães AAS, Lopes DS. Abordagens cirúrgicas para o tratamento das recessões marginais gengivais. Anais XV Congresso Brasileiro de Estomatologia. São Pedro- SP. 2007.
11. Shibayama R, Fugii WM. Enxerto gengival livre. UNOPAR Cient Ciênc Biol Saúde. 2000;2(1):107-11.

12. Andrade FSS, Clark RMO, Ferreira ML. Efeitos da laserterapia de baixa potência na cicatrização de feridas cutâneas. Rev Col Bras Cir. 2014;41(2):129-33.

13. Rocha JCT. Terapia laser, cicatrização tecidual e angiogênese. Rev Bras Promoção Saúde. 2004;17(1):44-8.

\section{CONFLITO DE INTERESSES}

Os autores declaram não haver conflitos de interesse.

\section{AUTOR PARA CORRESPONDÊNCIA}

\section{Matheus Francisco Barros Rodrigues}

matheusfbr08@outlook.com

Submetido em 27/03/2020

Aceito em 13/05/2020 